\title{
UNLOADING NEAR A SHEAR BAND IN GRANULAR MATERIAL
}

\author{
BY \\ MICHAEL SHEARER (North Carolina State University, Raleigh, North Carolina) \\ AND
}

DAVID G. SCHAEFFER (Duke University, Durham, North Carolina)

1. Introduction. We consider a model [4] for dynamic deformations of granular materials which allows for the localization of flow and the consequent development of shear bands. We construct solutions, asymptotic in small time, of Riemann initialvalue problems in one space dimension that include a shear band. An unusual feature of the solutions is that they are not scale invariant. The overall structure of the solution (shown in Figures 5 and 6 ) is that the material unloads elastically between the shear band and a free boundary that propagates into a region of plastic deformation. Mathematically, the Riemann problem reduces to solving a free boundary problem for the (linear) wave equation.

In the simplest case, which we call the symmetric case, the free boundary problem becomes a Goursat-type problem:

$$
\begin{aligned}
& \partial_{t} v=\partial_{x} \sigma \\
& \partial_{t} \sigma=c^{2} \partial_{x} v
\end{aligned}
$$

in a planar domain

$$
\{(x, t): t>0,0<x<s(t)\}
$$

bounded on the right by an unknown curve $\{x=s(t)\}$ emanating from the origin tangent to the $t$-axis. At the free boundary, (1.1) is subject to two boundary conditions of Dirichlet type:

$$
\begin{aligned}
& v(s(t), t)=\hat{v}(s(t) / t), \\
& \sigma(s(t), t)=\hat{\sigma}(s(t) / t)
\end{aligned}
$$

where $\hat{v}$ and $\hat{\sigma}$ are given functions. At $\{x=0\}$, system (1.1) is subject to a nonlinear, integral condition

$$
\sigma(0, t)=b\left(\frac{k}{\delta} \int_{0}^{t} v\left(0, t^{\prime}\right) d t^{\prime}\right)
$$

Received August 18, 1992.

1991 Mathematics Subject Classification. Primary 35L67, 73E99.

The first author's research was supported by National Science Foundation grant DMS 920115, which includes funds from AFOSR, and by Army Research Office grant DAAH04-94-G-0043.

The second author's research was supported by National Science Foundation grant DMS 9201034, which includes funds from AFOSR. 
where $b$ is a given function, $k$ is a dimensionless constant, and $\delta$ is a parameter (having units of length) related to the grain diameter. Since the domain (1.2) meets the $x$-axis only at the origin, no initial condition is needed. Compatibility of the data (1.3b) and (1.4) at the origin is achieved by ensuring that $b(0)=\hat{\sigma}(0)$.

Physically, $v$ represents a scalar velocity and $\sigma$ one component of the stress in the shearing of a one-dimensional continuum. The shear band is located at $x=0$, and (1.4) specifies its evolution. Unloading, governed by linear elasticity (1.1), occurs in the unknown region (1.2) adjacent to the shear band. The solution in the unloading region must match continuously a plastic rarefaction wave $\hat{v}(x / t), \hat{\sigma}(x / t)$ located to the right of (1.2); this is the content of (1.3).

In Sec. 2, we formulate the Riemann problems to be considered. In Sec. 3, we summarize and extend results of Garaizar [1] concerning these Riemann problems. The extension relates to the case in which a shear band forms. In this case, we find the structure of rarefaction waves is more degenerate than in the interior of the well-posed regime. Specifically, we derive an expansion of the functions $\hat{v}(\xi), \hat{\sigma}(\xi)$ around $\xi=0$. In Sec. 4, we begin the detailed discussion of solutions of Riemann problems with shear bands. We classify Riemann problems with no classical solution into three cases: the symmetric case and two nonsymmetric cases. We present equations in each case for the structure of nonclassical solutions that include a shear band. This section also includes the derivation of the boundary condition (1.4) at the shear band. In Sec. 5 , in which we restrict ourselves to the symmetric case, we find a series expansion of the solution about the point of initiation of the shear band. In the appendix, we derive the partial differential equations considered here from the model proposed in [4].

In a related paper [5], we prove short-time existence of a solution of the Riemann problem in the symmetric case. In a forthcoming paper [2], Garaizar and Schaeffer establish global existence of the solution. Their analysis is based on extending the solution examined with asymptotics here. They also present numerical results incorporating some of the ideas of this paper.

2. Statement of problem. We are concerned with Riemann problems for the following system of equations in one space variable $x$ and time $t$ :

$$
\begin{aligned}
\partial_{t} v & =\partial_{x} \sigma \\
\partial_{t} \sigma+\frac{1}{h(\gamma)} \partial_{t} \gamma & =c^{2} \partial_{x} v \\
\partial_{t} \tau-\frac{\alpha}{h(\gamma)} \partial_{t} \gamma & =0 \\
\partial_{t} \gamma & = \begin{cases}\partial_{t}(\sigma+\alpha \tau) & \text { if loading, } \\
0 & \text { if unloading, }\end{cases}
\end{aligned}
$$

where $h(\gamma)$ is a positive decreasing function on an interval containing the point $\gamma=0$ and

$$
h(0)=\alpha^{2} .
$$

In these equations, the parameter $c$ is the elastic wave speed and the parameter $\alpha$ measures the degree of nonassociativity in the model. The unknowns are $v$, the 
velocity; $\sigma$ and $\tau$, components of stress; and $\gamma$, the yield stress. The variables are required to satisfy the inequality $\sigma+\alpha \tau \leq \gamma$, which is called the yield condition. In (2.1), loading means $\gamma=\sigma+\alpha \tau$, and $\partial_{t}(\sigma+\alpha \tau)>0$. In unloading, $\sigma+\alpha \tau \leq \gamma$, and the equations are those of linear elasticity. We rewrite them here for future reference:

$$
\begin{aligned}
& \partial_{t} v=\partial_{x} \sigma, \\
& \partial_{t} \sigma=c^{2} \partial_{x} v, \\
& \partial_{t} \tau=0, \\
& \partial_{t} \gamma=0 .
\end{aligned}
$$

The Riemann problem for system (2.1) is the initial-value problem with initial data of the form

$$
(v, \sigma, \tau, \gamma)(x, 0)= \begin{cases}\left(v_{L}, \sigma_{L}, \tau_{L}, \gamma_{L}\right) & \text { if } x<0, \\ \left(v_{R}, \sigma_{R}, \tau_{R}, \gamma_{R}\right) & \text { if } x>0,\end{cases}
$$

subject to $(\sigma+\alpha \tau)(x, 0) \leq \gamma(x, 0)$.

In solving Riemann problems for system (2.1), Garaizar [1] found initial data (2.4) for which there is no scale-invariant solution. The reason is that Eqs. (2.1) lose hyperbolicity at $\gamma=0$ and the classical construction of scale-invariant solutions breaks down.

When the equations lose hyperbolicity, they also lose linear well-posedness, and we suppose that a shear band forms. As in [4], we treat the shear band as a stationary discontinuity, with nonstandard jump conditions. Specifically, the velocity gradient is approximated by a divided difference:

$$
v_{x} \approx[v] / \delta
$$

where $[v]$ is the jump in velocity across the shear band and $\delta$ is a small parameter measuring the thickness of the shear band. From the conservation of momentum equation (2.1a), we see that $\sigma$ is continuous across a shear band. The variable $\tau$ experiences a jump on each side of the shear band and is modelled within the shear band by an internal variable $z$. The approximation (2.5) leads to the following system of ordinary differential equations, with a constraint, for evolution of the variables $\sigma, z, \gamma$ within the shear band:

$$
\begin{aligned}
\partial_{t} \sigma+\frac{1}{h(\gamma)} \partial_{t} \gamma & =c^{2} \frac{[v]}{\delta}, \\
\partial_{t} z-\frac{\alpha}{h(\gamma)} \partial_{t} \gamma & =0, \\
\gamma & =\sigma+\alpha z .
\end{aligned}
$$

Note that these equations are coupled to the external variables through the jump [ $v$ ] in $v$ and through the continuity of $\sigma$. This system of equations may be regarded as a jump condition for stationary shocks, analogous to the usual Rankine-Hugoniot condition for shocks. However, the Rankine-Hugoniot condition is a system of algebraic equations, in contrast to (2.6), which is a system of differential equations. The jump condition (2.6) effectively widens the class of weak solutions of equations (2.1) beyond the class of solutions whose jump discontinuities satisfy the usual 
Rankine-Hugoniot conditions. It is within this wider class that we shall seek solutions of Riemann problems that have no classical solution. Note that system (2.6) is not scale invariant, because of the right-hand side of equation (2.6a). Correspondingly, solutions of Riemann problems with no classical solution also fail to be scale invariant.

3. Riemann problems without shear bands. In this section, we review and extend some results of Garaizar [1] concerning Riemann problems for system (2.1). In Subsection 3.1 we summarize characteristic information, which is used in Subsection 3.2 to describe rarefaction waves. We also derive asymptotic forms of rarefaction waves near the point $\gamma=0$ at which a shear band is initiated. In Subsection 3.3 we describe the construction of wave curves used to solve Riemann problems.

3.1. Characteristic information. Let $U=(v, \sigma, \tau)^{\mathrm{T}}$, and write system $(2.1 \mathrm{a}, \mathrm{b}$, c) in the loading case (in which $\gamma=\sigma+\alpha \tau$ ) in the form

$$
U_{t}+B U_{x}=0
$$

where

$$
B=-\frac{1}{h-\alpha^{2}+1}\left(\begin{array}{ccc}
0 & h-\alpha^{2}+1 & 0 \\
c^{2}\left(h-\alpha^{2}\right) & 0 & 0 \\
\alpha c^{2} & 0 & 0
\end{array}\right) .
$$

Characteristic speeds of (3.1) are eigenvalues of $B$, given by

$$
\lambda_{ \pm}= \pm c \sqrt{\eta}, \quad \lambda_{0}=0,
$$

where $\eta=\left(h-\alpha^{2}\right) /\left(h-\alpha^{2}+1\right)$. The associated eigenvectors are (respectively)

$$
r_{ \pm}=\left(c^{-1} \sqrt{\eta}, \mp \eta, \mp \alpha(1-\eta)\right)^{\mathrm{T}}, \quad r_{0}=(0,0,1)^{\mathrm{T}} .
$$

We conclude that system (3.1) is hyperbolic if and only if $\eta \geq 0$. Recall that $h=h(\gamma)$ is a decreasing function with $h(0)=\alpha^{2}$. Therefore, (3.1) is hyperbolic if and only if $\gamma \leq 0$. For simplicity, we shall assume that $h$ is strictly decreasing, in symbols

$$
h^{\prime}(\gamma)<0,
$$

as this will be helpful later. In particular, (3.5) implies that system (3.1) is genuinely nonlinear for $\gamma<0$.

REMARK. Condition (3.5) rules out shocks during loading if we impose the Lax entropy condition [3] (see [1]). To see this, consider a shock wave across which the material is loading. Then (3.5) implies that $\eta$, hence the characteristic speed, is decreasing across the shock. This violates the Lax entropy condition, which requires the shock speed to be between the characteristic speeds on either side of the shock. We conclude that the only physical loading waves are rarefaction waves.

3.2. Rarefaction waves. In part (a) of this subsection, we derive formulae for the variables in a rarefaction wave (cf. also [1]). In part (b), we obtain the dependence of the variables in a rarefaction wave near the point $\gamma=0$ where the equations lose hyperbolicity.

(a) Centered rarefaction waves are continuous expansion waves of the scale-invariant form $U=U_{ \pm}(x / t)$. In order to be solutions of the loading equations (2.1), the 
functions $U_{ \pm}(x / t)$ must take values on integral curves $R_{ \pm}$of the eigenvectors $r_{ \pm}$ (respectively), with $x / t=\lambda_{ \pm}$. Let $\xi=x / t$. Since $\lambda_{ \pm}= \pm c \sqrt{\eta}$ depends only on $\gamma$, we obtain an equation relating $\xi$ and $\gamma$ in a rarefaction wave:

$$
\xi= \pm c \sqrt{\frac{h(\gamma)-\alpha^{2}}{h(\gamma)-\alpha^{2}+1}} .
$$

Let $k(\gamma)=h(\gamma)-\alpha^{2}$, and rearrange (3.6) as

$$
k(\gamma)=\frac{\xi^{2}}{c^{2}-\xi^{2}} .
$$

Now $k(0)=h(0)-\alpha^{2}=0$ and $k^{\prime}(\gamma)<0$. Recall that the domain of $h$, and hence of $k$, is an interval $\left[\gamma_{1}, \gamma_{2}\right]$ with $\gamma_{1}<0<\gamma_{2}$. Therefore, $k$ is an invertible function from $\left[\gamma_{1}, 0\right]$ to $\left[0, k\left(\gamma_{1}\right)\right]$. Moreover, the right-hand side of (3.7) is monotonically increasing as a function of $\xi^{2}, 0 \leq \xi^{2}<c^{2}$, and is onto $[0, \infty)$. Therefore, Eq. (3.7) has a unique solution $\gamma=\hat{\gamma}(\xi)$ for all $\xi$ such that $\xi^{2} \leq k\left(\gamma_{1}\right)=h\left(\gamma_{1}\right)-\alpha^{2}$. Moreover, since the inverse of $k$ is analytic, $\hat{\gamma}(\xi)$ is an analytic function of $\xi^{2}$. Note that $\hat{\gamma}(\xi)$ is a decreasing function of $\xi^{2}$. It is not hard to derive the following parameterization of the integral curves $R_{ \pm}$by $\gamma=\sigma+\alpha \tau$ :

$$
\begin{aligned}
& \frac{d v}{d \gamma}=\mp \frac{1}{\operatorname{ch}(\gamma)} \sqrt{k(\gamma)(k(\gamma)+1)}, \\
& \frac{d \sigma}{d \gamma}=1-\frac{\alpha^{2}}{h(\gamma)}, \\
& \frac{d \tau}{d \gamma}=\frac{\alpha}{h(\gamma)} .
\end{aligned}
$$

Therefore, we have the representation of rarefaction waves:

$$
\begin{aligned}
& v= \begin{cases}\hat{v}_{r}(\xi)=v_{0}-\int_{\gamma_{0}}^{\hat{\gamma}} \frac{1}{\operatorname{ch}(\theta)}[k(\theta)(k(\theta)+1)]^{1 / 2} d \theta & \text { on } R_{+}, \\
\hat{v}_{l}(\xi)=v_{0}+\int_{\gamma_{0}}^{\hat{\gamma}} \frac{1}{\operatorname{ch}(\theta)}[k(\theta)(k(\theta)+1)]^{1 / 2} d \theta & \text { on } R_{-} ;\end{cases} \\
& \sigma=\hat{\sigma}(\xi)=\sigma_{0}+\hat{\gamma}-\gamma_{0}-\alpha^{2}\left(H(\hat{\gamma})-H\left(\gamma_{0}\right)\right) ; \\
& \tau=\hat{\tau}(\xi)=\tau_{0}+\alpha\left(H(\hat{\gamma})-H\left(\gamma_{0}\right)\right),
\end{aligned}
$$

where

$$
H(\gamma)=\int_{0}^{\gamma} \frac{1}{h(\theta)} d \theta
$$

for notational convenience in (3.9) we have suppressed the dependence of $\hat{\gamma}$ on $\xi$. In order that $\gamma$ be increasing in time, we have $\gamma \leq \gamma_{0} \leq 0$ if $\gamma_{0}$ is the value of $\gamma$ at the trailing edge of the rarefaction wave.

(b) Next, we describe the structure of these rarefaction waves near the end points of the integral curves $R_{ \pm}$, where $\gamma=0$. Consider a right-moving rarefaction wave whose trailing edge has speed zero, and let $v_{0}, \sigma_{0}, \tau_{0}=-\alpha^{-1} \sigma_{0}, \gamma_{0}=0$ be the values of the variables at $\xi=0$. In the following lemma, we find expressions for the highest-order dependence of $\hat{v}_{r}, \hat{\sigma}, \hat{\tau}, \hat{\gamma}$ on $\xi$ near $\xi=0$. (Almost identical expressions apply to left-moving rarefaction waves.) 
LemMA 3.1. Suppose $h(\gamma)$ is real analytic in a neighborhood of $\gamma=0, h(0)=\alpha^{2} \neq$ 0 , and $h^{\prime}(0)=-h_{1}<0$. Then

$$
\begin{aligned}
& \hat{\gamma}(\xi)=-\frac{\xi^{2}}{c^{2} h_{1}}+\xi^{4} f_{1}\left(\xi^{2}\right), \\
& \hat{v}_{r}(\xi)=v_{0}+v_{3} \xi^{3}+\xi^{5} f_{2}\left(\xi^{2}\right), \\
& \hat{\sigma}(\xi)=\sigma_{0}-\sigma_{4} \xi^{4}+\xi^{6} f_{3}\left(\xi^{2}\right), \\
& \hat{\tau}(\xi)=\tau_{0}-\frac{1}{\alpha h_{1} c^{2}} \xi^{2}+\xi^{4} f_{4}\left(\xi^{2}\right),
\end{aligned}
$$

where

$$
v_{3}=\frac{2}{3 \alpha^{2} h_{1} c^{4}}, \quad \sigma_{4}=\frac{1}{2 \alpha^{2} h_{1} c^{4}},
$$

and the functions $f_{i}, i=1, \ldots, 4$, are real analytic near the origin.

Proof. By previous assumptions on $h$, we have

$$
h(\gamma)=\alpha^{2}-h_{1} \gamma+O\left(\gamma^{2}\right),
$$

where $h_{1}=-h^{\prime}(0)>0$. From (3.7),

$$
-h_{1} \gamma+O\left(\gamma^{2}\right)=\frac{\xi^{2}}{c^{2}}\left(1-\frac{\xi^{2}}{c^{2}}\right)^{-1}=\sum_{n=1}^{\infty}\left(\frac{\xi^{2}}{c^{2}}\right)^{n} .
$$

Therefore, if

$$
\gamma=\hat{\gamma}(\xi)=\sum_{n=0}^{\infty} \gamma_{2 n} \xi^{2 n}
$$

we have

$$
\gamma_{0}=0, \quad \gamma_{2}=-\frac{1}{c^{2} h_{1}}
$$

which confirms $(3.11 \mathrm{a})$.

The properties of $\hat{\sigma}(\xi)$ are easily verified; so we treat $\hat{\sigma}$ next. Since $h$ is analytic and $h(0)=\alpha^{2} \neq 0$, the function $1 / h(\theta)$ is analytic for $\theta$ near zero. Thus $\hat{\sigma}$ given in (3.9) is an analytic function of $\gamma$, which is in turn an analytic function of $\xi^{2}$. Moreover, $d \hat{\sigma} / d \gamma=1-\alpha^{2} / h(\gamma)=0$ at $\gamma=0$, which implies that the coefficient of $\xi^{2}$ in the expansion of $\hat{\sigma}(\xi)$ is zero.

The form of $\hat{v}_{r}(\xi)$ is somewhat more complicated. The dependence of $\hat{v}_{r}$ on $\gamma$ in (3.9) would be analytic were it not for the term $\sqrt{k(\theta)}$. Now $k(0)=h(0)-\alpha^{2}=0$, so that

$$
k(\theta)=\theta K(\theta),
$$

where $K$ is the analytic function defined by

$$
K(\theta)= \begin{cases}\theta^{-1}\left(h(\theta)-\alpha^{2}\right) & \text { if } \theta \neq 0 \\ -h_{1} & \text { if } \theta=0\end{cases}
$$


In particular, $K(0)<0$. Therefore, as a function of $\gamma \leq 0$, we have

$$
\hat{v}_{r}=v_{0}-\int_{0}^{\hat{\gamma}} f(\theta) \sqrt{-\theta} d \theta
$$

where $f(\theta)=[-K(\theta)(k(\theta)+1)]^{1 / 2} / \operatorname{ch}(\theta)$ is analytic in a neighborhood of $\theta=0$. Changing variables in (3.19), we find

$$
\hat{v}_{r}=v_{0}+2 \int_{0}^{\sqrt{-\gamma}} f\left(-u^{2}\right) u^{2} d u
$$

Since the integrand in (3.20) is an even analytic function of $u$ that is at least quadratic at $u=0$, we deduce that

$$
\hat{v}_{r}=v_{0}+(-\hat{\gamma})^{3 / 2} g(\hat{\gamma})
$$

where $g$ is analytic. Now using the form (3.11a) for $\gamma=\hat{\gamma}(\xi)$, we conclude that $\hat{v}_{r}(\xi)$ has the expansion $(3.11 \mathrm{~b})$. The formulae (3.12) for the coefficients $v_{3}, \sigma_{4}$ are easy to establish from (3.9), (3.11a).

The argument for $\hat{\tau}$, including the derivation of formula (3.11d), is similar to that for $\hat{\sigma}$. This completes the proof.

3.3. Wave curves. To solve Riemann problems, we need to consider centered solutions of system (2.1), including solutions of the unloading equations (2.3). Since the latter equations are linear, their centered wave solutions are shocks, moving with one of the characteristic speeds $\pm c$ or 0 . For moving shocks, the Rankine-Hugoniot conditions reduce to

$$
[\sigma] \pm c[v]=0, \quad[\tau]=[\gamma]=0
$$

along $x= \pm c t$ respectively. Across stationary shocks, $v$ and $\sigma$ are continuous while $\tau$ and $\gamma$ may experience arbitrary jumps (subject to the yield condition $\sigma+\alpha \tau \leq \gamma$ ).

As explained in [1], unloading shocks and loading rarefaction waves may be combined to form groups of waves, one group moving to the right and a second group moving in turn to the left. We begin by studying groups of right-moving waves. Such a wave group is shown in Figure 1 on p. 586. Here, $U=(v, \sigma, \tau, \gamma)^{\mathrm{T}}$. The constant values $U_{N}, U_{R}$ are related by the Rankine-Hugoniot conditions (3.22) and the condition that $U_{N}$ is at yield, i.e., $\gamma_{N}=\sigma_{N}+\alpha \tau_{N}$. The other state at yield, $U_{0}$, is related to $U_{N}$ by the fact that they are separated by a rarefaction wave. Therefore, $\left(v_{0}, \sigma_{0}, \tau_{0}\right)$ and $\left(v_{N}, \sigma_{N}, \tau_{N}\right)$ lie on the same integral curve $R_{+}$.

Note that this wave group solves a quarter-plane problem, with boundary and initial conditions

$$
\begin{aligned}
U(x, 0) & =U_{R}, & & x>0, \\
U(0, t) & =U_{0}, & & t>0,
\end{aligned}
$$

where the data satisfy the restrictions

$$
\sigma_{R}+\alpha \tau_{R} \leq \gamma_{R} \leq \gamma_{0}=\sigma_{0}+\alpha \tau_{0} .
$$

For a given $U_{R}$, the quarter-plane problem cannot be solved for arbitrary data $U_{0}$ because the problem would be overdetermined. In fact, for each $U_{R}$, there is a wave curve $W_{+}\left(U_{R}\right)$ of values of $U_{0}$ for which the quarter-plane problem has a solution. 


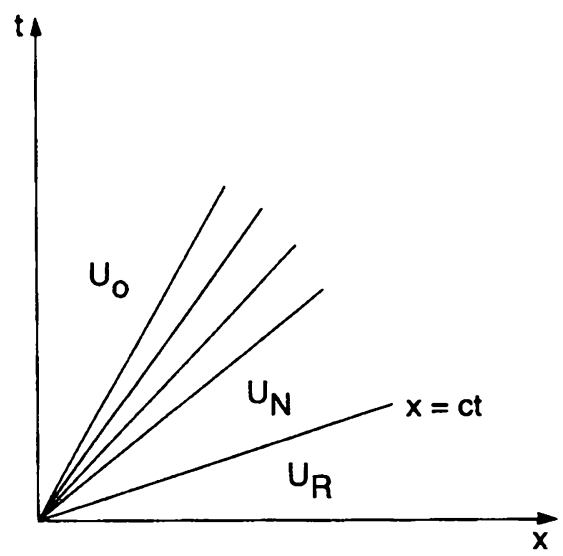

Fig. 1. Right-moving wave group

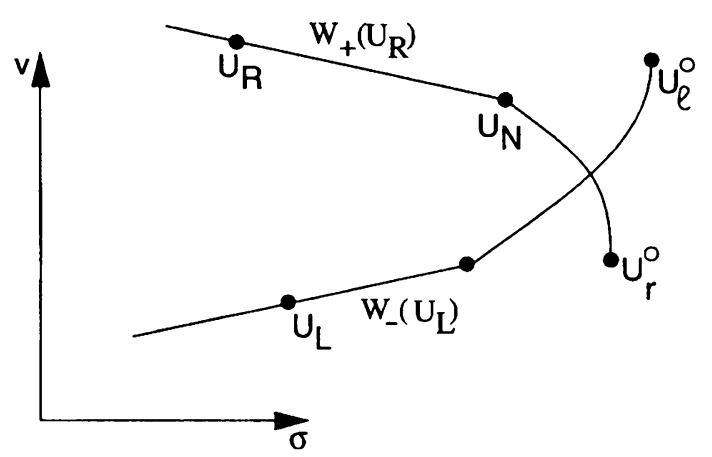

FIG. 2. Wave curves

The projection onto the $(\sigma, v)$-plane of a typical wave curve is shown in Figure 2. Note that there is a curved section, corresponding to a wave group as in Figure 1, and a straight section, corresponding to a single elastic shock separating $U_{R}$ and $U_{0}$.

First consider solutions of the quarter-plane problem involving a single elastic shock. For fixed $U_{R}$, the corresponding states $U_{0}$ are represented by the straight line from (3.22):

$$
v-v_{R}=-c\left(\sigma-\sigma_{R}\right)
$$

in the $(\sigma, v)$-plane, with $\tau$ and $\gamma$ constant, and $\sigma+\alpha \tau \leq \gamma$.

Next, we construct the curved portion of $W_{+}\left(U_{R}\right)$, referring to Figures 1 and 2. Consider $U_{R}$ fixed. The state $U_{N}$ in Figures 1 and 2 lies at the end of the straight portion of $W_{+}\left(U_{R}\right)$ and is determined by $U_{R}$, i.e.,

$$
\begin{aligned}
& v_{N}=v_{R}-c\left(\gamma_{R}-\alpha \tau_{R}-\sigma_{R}\right), \\
& \sigma_{N}=\gamma_{R}-\alpha \tau_{R}, \\
& \tau_{N}=\tau_{R}, \\
& \gamma_{N}=\gamma_{R} .
\end{aligned}
$$

Then $U_{0}$ is restricted to lie on the integral curve $R_{+}$through $U_{N}$, with the condition $\gamma_{0} \geq \gamma_{N}$. For fixed $U_{R}$, we can represent the family of $U_{0}$ by a curve in the $(\sigma, v)$ plane, namely, the projection of the integral curve $R_{+}$through $U_{N}$, retaining only 
the portion with $\gamma \geq \gamma_{N}$. Finally, if $U_{R}$ is itself at yield, then there can be a plastic rarefaction alone, with $U_{0}$ lying on the integral curve $R_{+}$through $U_{R}$. That is, $U_{N}=U_{R}$ in Figures 1 and 2.

Putting these solutions and the wave curves together, we obtain the wave curve $W_{+}\left(U_{R}\right)$ in the $(\sigma, v)$-plane shown in Figure 2, along which $v$ is a monotonically decreasing function of $\sigma$. This wave curve represents all data $U_{0}$ for which the quarter-plane problem (3.23) has a solution. Similarly, we construct a wave curve $W_{-}\left(U_{L}\right)$. This is the projection onto the $(\sigma, v)$-plane of the set of all $U_{0}$ for which the quarter-plane problem in the second quadrant with boundary conditions

$$
\begin{aligned}
U(x, 0) & =U_{L}, & & x<0, \\
U(0, t) & =U_{0}, & & t>0,
\end{aligned}
$$

has a centered solution. This is also shown in Figure 2 for a specific $U_{L}$. Note that $v$ is a monotonically increasing function of $\sigma$ along $W_{-}\left(U_{L}\right)$.

A point of intersection of $W_{-}\left(U_{L}\right), W_{+}\left(U_{R}\right)$ (and by monotonicity there is at most one such point) corresponds to a classical solution of the Riemann problem. In this solution, the variables $\sigma, v$ are continuous across the $t$-axis (with their values given by the point of intersection of $\left.W_{-}\left(U_{L}\right), W_{+}\left(U_{R}\right)\right)$, while $\gamma$ and $\tau$ experience a jump.

The real interest of this paper is in values of $U_{L}, U_{R}$ for which $W_{-}\left(U_{L}\right), W_{+}\left(U_{R}\right)$ do not intersect. This occurs because the wave curves necessarily terminate at $\gamma=0$. Although we have shown data $U_{L}, U_{R}$ in Figure 2 for which the wave curves do intersect, we can increase $v_{R}$ or decrease $v_{L}$ so that $W_{-}\left(U_{L}\right), W_{+}\left(U_{R}\right)$ do not intersect (cf. Figure 3). In this case, there is no classical scale-invariant solution of the Riemann problem.

4. Solutions with shear bands. We are interested in values of $U_{L}, U_{R}$ for which $W_{-}\left(U_{L}\right)$ and $W_{+}\left(U_{R}\right)$ do not intersect. In this circumstance, we seek a solution with a shear band. In Subsection 4.1, we reformulate the boundary condition at the shear band by integrating system (2.6) for the evolution of the variables in the shear band. In Subsection 4.2, we classify Riemann problems into three cases according to the structure of the solution. In Subsection 4.3, we derive the equations to be solved in each case.

4.1. Derivation of boundary condition at a shear band. In this subsection, we integrate the equations (2.6) to obtain a relation of the form (1.4) between $\sigma$ and the jump $[v]$ in $v$ across the shear band.

LEMMA 4.1. Suppose $h(\gamma)$ is real analytic in a neighborhood of $\gamma=0, h(0)=$ $\alpha^{2} \neq 0$, and $h^{\prime}(0)=-h_{1}<0$. Let $(\sigma, z, \gamma,[v])(t)$ satisfy equations (2.6), and let $(\sigma, z, \gamma)(0)=\left(\sigma_{0},-\sigma_{0} / \alpha, 0\right)$ for some $\sigma_{0}$. Then there is a function $b=b(\gamma)$ that is real analytic in a neighborhood of $\gamma=0$ such that

$$
b(0)=\sigma_{0}, \quad b^{\prime}(0)=0, \quad b^{\prime \prime}(0)=-\alpha^{2} / h_{1}
$$

and with the property that

$$
\sigma(t)=b\left(\frac{c^{2} h_{1}}{\delta} \int_{0}^{t}[v](\theta) d \theta\right) .
$$


Proof. Let

$$
J(t)=c^{2} \delta^{-1} \int_{0}^{t}[v](\theta) d \theta, \quad H(\gamma)=\int_{0}^{\gamma} \frac{1}{h(y)} d y .
$$

Then from (2.6),

$$
\sigma+H(\gamma)=J+\sigma_{0} \quad \text { and } \quad z-\alpha H(\gamma)=-\sigma_{0} / \alpha, \quad \gamma=\sigma+\alpha z
$$

From (4.4) we obtain

$$
\begin{gathered}
\sigma+\alpha^{-1} z=J+\sigma_{0}\left(1-\alpha^{-2}\right), \\
\gamma+\left(1-\alpha^{2}\right) H(\gamma)=J .
\end{gathered}
$$

Let $F(\gamma)$ denote the left-hand side of (4.6). Then $\gamma=F^{-1}(J)$, i.e.,

$$
\sigma+\alpha z=F^{-1}(J) \text {. }
$$

Now solving (4.5), (4.7) for $\sigma$, we obtain

$$
\sigma=\hat{b}(J)
$$

where

$$
\hat{b}(J)=\sigma_{0}+\left(1-\alpha^{2}\right)^{-1}\left\{F^{-1}(J)-\alpha^{2} J\right\} .
$$

From the hypotheses on $h$, we see that $F(\gamma)$ is analytic near $\gamma=0$. Moreover,

$$
F(0)=0, \quad F^{\prime}(0)=\alpha^{-2}, \quad F^{\prime \prime}(0)=\left(1-\alpha^{2}\right) h_{1} / \alpha^{4},
$$

where $h_{1}=-h^{\prime}(0)>0$. By the inverse function theorem, $\hat{b}(J)$ is analytic near $J=0$. Let $b(y)=\hat{b}\left(y / h_{1}\right)$. Then (4.1), (4.2) follow easily from (4.8)-(4.10). This completes the proof.

In Eqs. (2.6), we might regard $[v]$ as a given function of time: $[v]=[v](t)$. We could then integrate (2.6), with the given initial conditions for $\sigma, z, \gamma$. The lemma gives a formula for how the solution component $\sigma(t)$ would depend on $[v](t)$. Note that the combination $c^{2} h_{1}$ is nondimensional, so that the argument of $b$ is nondimensional; $b$ itself has the dimensions of stress.

4.2. Form of the solutions. Now we are ready to consider the central problem. Consider the Riemann problem with initial data

$$
U(x, 0)= \begin{cases}U_{L} & \text { if } x<0 \\ U_{R} & \text { if } x>0\end{cases}
$$

for which there is no classical centered solution of system (2.1). Let $\left(\sigma_{l}^{0}, v_{l}^{0}\right),\left(\sigma_{r}^{0}, v_{r}^{0}\right)$ denote the end points of the wave curves $W_{-}\left(U_{L}\right), W_{+}\left(U_{R}\right)$, respectively. We distinguish three cases, depending on the relative positions of these points. The three cases are illustrated in Figure 3.

(a) Symmetric case. $\sigma_{l}^{0}=\sigma_{r}^{0} ; v_{r}^{0}>v_{l}^{0}$.

(b) Nonsymmetric case with rarefactions. Here, $\sigma_{l}^{0}>\sigma_{r}^{0}$ and $v_{r}^{0}>\bar{v}_{l}$, where $\bar{v}_{l}$ is the value of $v$ such that $\left(\sigma_{r}^{0}, \bar{v}_{l}\right) \in W_{-}\left(U_{L}\right)$. In this case, we assume $\left(\sigma_{r}^{0}, \bar{v}_{l}\right)$ 


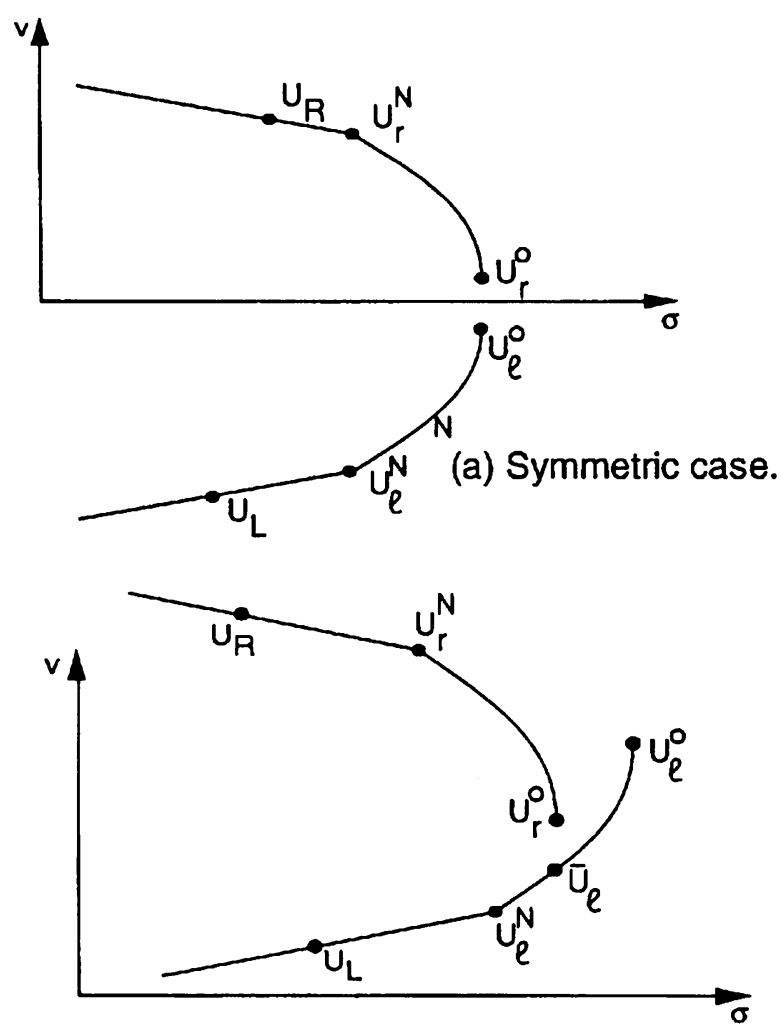

(b) Nonsymmetric case: Two rarefactions.

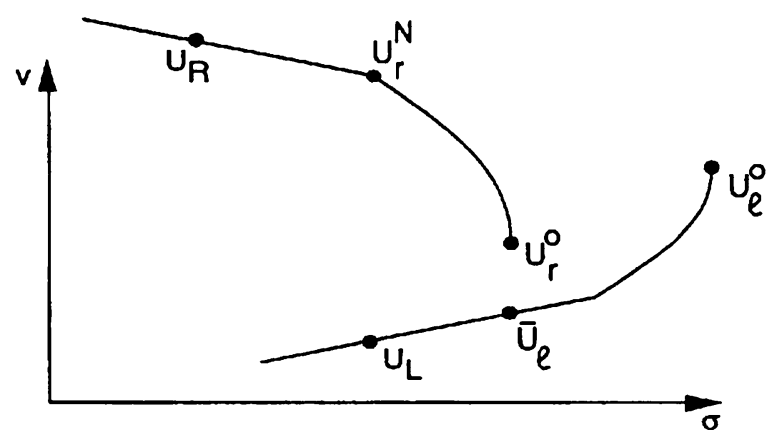

(c) Nonsymmetric case: Rarefaction and shock.

FIG. 3. Three cases of Riemann problems with no classical solution

lies on the curved portion of $W_{-}\left(U_{L}\right)$ so that $\left(\sigma_{r}^{0}, \bar{v}_{l}\right)$ is the value of $(\sigma, v)$ on the trailing edge of a rarefaction wave, that is, $\left(\sigma_{r}^{0}, \bar{v}_{l}\right)=\left(\hat{\sigma}_{l}(a), \hat{v}_{l}(a)\right)$ for some $a<0$.

(c) Nonsymmetric case with shock wave. This is the same as case (b) except $\left(\sigma_{r}^{0}, \bar{v}_{l}\right)$ is on the straight part of $W_{-}\left(U_{L}\right)$.

REMARK. There are also nonsymmetric cases similar to cases (b) and (c) but with $\sigma_{l}^{0}<\sigma_{r}^{0}$. These cases can be obtained from cases (b) and (c) by reversing left and right. 
Since there is no classical solution in each case, we explore the possibility of solving the initial-value problem by including a shear band. In such a solution, $v$ (in addition to $\tau$ and $\gamma$ ) can experience a jump across the $t$-axis. In contrast with the classical solution, the solution with a shear band is nonconstant on the $t$-axis because of Eqs. (2.6), and the overall solution does not enjoy the property of scale invariance. Here we discuss the form of the solution. In Sec. 5 we find the first few terms of an asymptotic series for the solution.

To start with, consider Eqs. (2.6) on the shear band, which is located on the $t$-axis. To understand the short-time behavior, we rescale time $t$ by a small constant: $t^{\prime}=$ $t / \varepsilon$. Then $\partial_{t}=\frac{1}{\varepsilon} \partial_{t^{\prime}}$, so that Eqs. (2.6) are unchanged apart from an $\varepsilon$ multiplying the right-hand side. Then if $\varepsilon$ is small compared with $\delta$, we effectively have scaleinvariant equations; viz.,

$$
\begin{aligned}
& \partial_{t^{\prime}} \sigma+\frac{1}{h(\gamma)} \partial_{t^{\prime}} \gamma=0, \\
& \partial_{t^{\prime}} \tau-\frac{\alpha}{h(\gamma)} \partial_{t^{\prime}} \gamma=0, \quad \gamma=\sigma+\alpha \tau .
\end{aligned}
$$

It follows that $\sigma, \tau$, and $\gamma$ are constant in time to leading order in $\varepsilon$. Thus for small time, specifically, for times that are small compared to $\delta$, the solution is approximately scale invariant. Therefore, it resembles the solution suggested by the wave curves of Figure 2. In this solution, there is a rarefaction wave on the right extending up to the $t$-axis, and on the left of the $t$-axis there will be either a shock or a rarefaction, depending on the case. These blown-up solutions are shown in Figure 4.

We modify this picture for larger times as follows. The solution is no longer scale invariant, and the material unloads away from the shear band. We therefore postulate an unloading or relief wave propagating away from the shear band and interacting with the rarefaction. In cases (a) and (b) where there is a rarefaction on each side of the shear band, there will also be a relief wave on each side of the shear band. In the remaining case (c), there will be a relief wave only on the right. Each relief wave should enter the origin tangent to the trailing edge of the rarefaction wave on the same side, so that there is agreement with Figure 4 as $t \rightarrow 0+$. The conjectured structure is shown in Figure 5 on p. 592.

In those figures, the solution is scale invariant outside the region bounded by the relief waves and agrees with the blown-up solution there. (In case (c), the single leftmoving wave, which is characteristic, plays the role of the relief wave.) We therefore have a pair of coupled quarter-plane problems to solve in the regions $E_{l}, E_{r}$ of Figure 5, with the boundaries in the $(x, t)$-plane being the two relief waves and the $t$-axis. Note that regions $E_{l}, E_{r}$ have $t<T$, where $T$ is chosen so that the relief wave has not completely penetrated the rarefaction by time $T$.

4.3. Equations for the solution. Let $E_{l}=\left\{s_{l}(t)<x<0,0<t<T\right\}$ and $E_{r}=\left\{0<x<s_{r}(t), 0<t<T\right\}$ be the regions shown in Figure 5. In these regions, the deformation is elastic. Thus, $\gamma$ and $\tau$ are constant in time in $E_{l}$ and $E_{r}$ and are therefore determined by their values on the moving boundaries. We are left with 


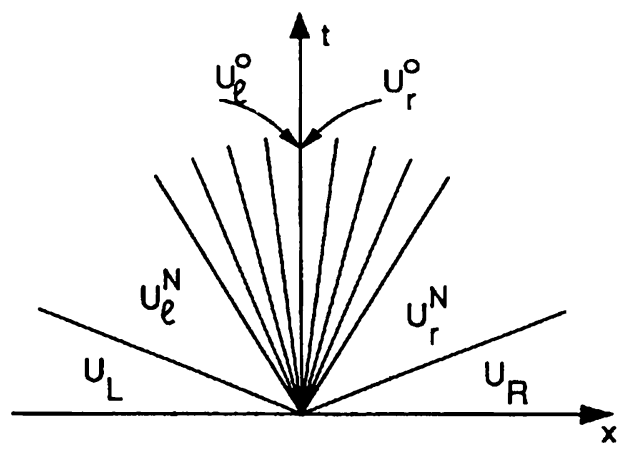

(a) Symmetric Case.

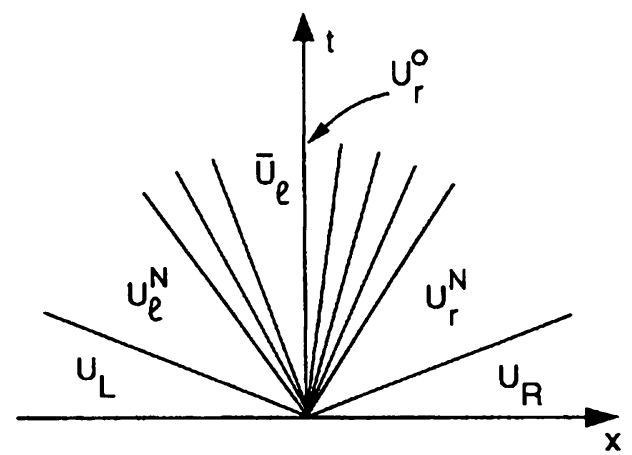

(b) Nonsymmetric Case, two rarefactions.

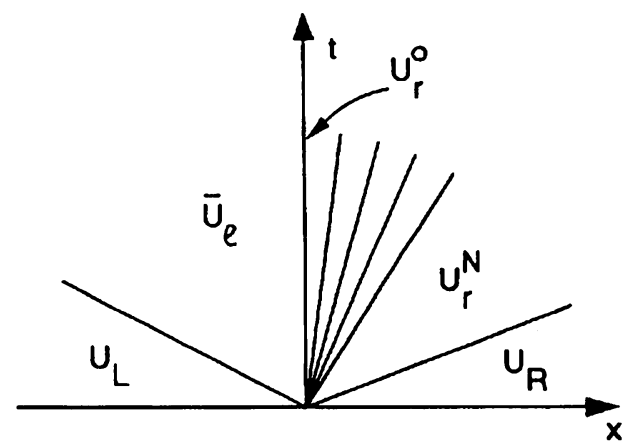

(c) Nonsymmetric case, shock and rarefaction.

Fig. 4. Blown-up solutions of Riemann problems

variables $v, \sigma$ satisfying the wave equation:

$$
\begin{aligned}
& \partial_{t} v=\partial_{x} \sigma, \\
& \partial_{t} \sigma=c^{2} \partial_{x} v .
\end{aligned}
$$

In what follows, we write down the general solution of Eq. (4.13). We then impose boundary conditions on the moving boundaries and boundary conditions on the $t$ axis dictated by the evolution of the variables in the shear band through Eqs. (2.6). 


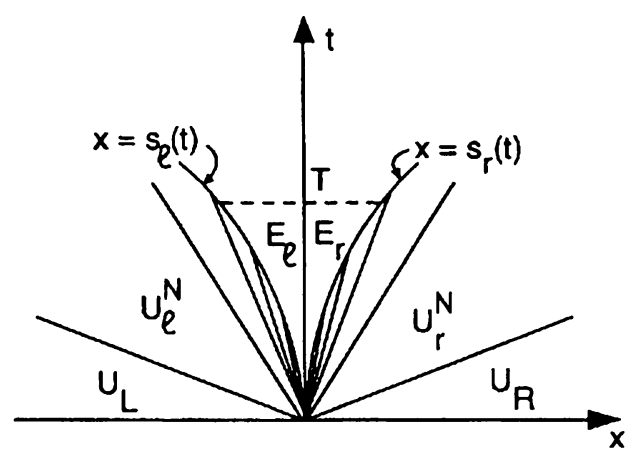

(a) Symmetric Case.

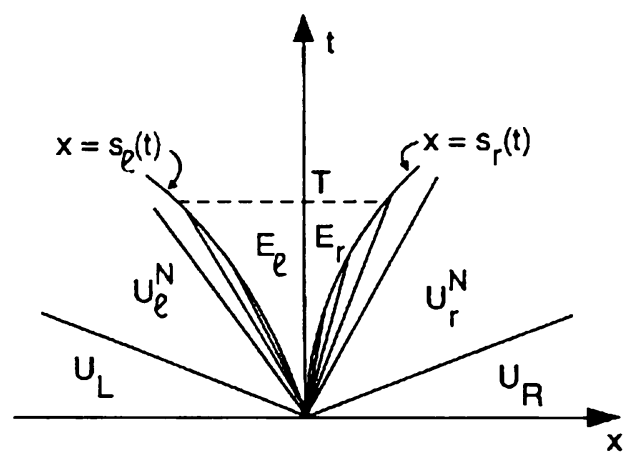

(b) Nonsymmetric Case, two rarefactions.

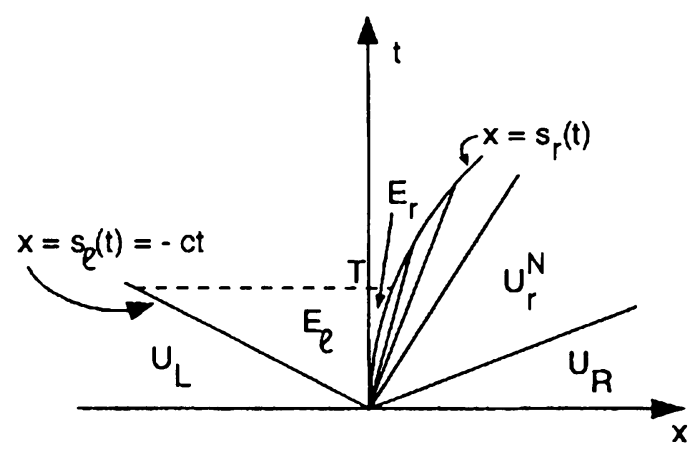

(c) Nonsymmetric case, shock and rarefaction.

Fig. 5. Solutions of Riemann problems

Let $\left(v_{l}, \sigma_{l}\right)(x, t)$ denote the solution in $E_{l}$, and let $\left(v_{r}, \sigma_{r}\right)$ denote the solution in $E_{r}$. Since $\left(v_{l}, \sigma_{l}\right),\left(v_{r}, \sigma_{r}\right)$ separately satisfy the wave equation (4.13), we have d'Alembert's solution, which we write in the form

$$
\begin{aligned}
& \left(\begin{array}{l}
v_{l} \\
\sigma_{l}
\end{array}\right)(x, t)=F_{l}(c t+x)\left(\begin{array}{l}
1 \\
c
\end{array}\right)+G_{l}(c t-x)\left(\begin{array}{c}
1 \\
-c
\end{array}\right), \quad s_{l}(t)<x<0, \\
& \left(\begin{array}{l}
v_{r} \\
\sigma_{r}
\end{array}\right)(x, t)=F_{r}(c t+x)\left(\begin{array}{l}
1 \\
c
\end{array}\right)+G_{r}(c t-x)\left(\begin{array}{c}
1 \\
-c
\end{array}\right), \quad 0<x<s_{r}(t) \text {. }
\end{aligned}
$$


Recalling the rarefaction waves of Subsection 3.2, we have boundary conditions for $v, \sigma$ along the relief wave located at $x=s_{r}(t)$ which relate $F_{r}, G_{r}, s_{r}$ as follows:

$$
\begin{aligned}
& F_{r}\left(c t+s_{r}(t)\right)+G_{r}\left(c t-s_{r}(t)\right)=\hat{v}_{r}\left(s_{r}(t) / t\right), \\
& F_{r}\left(c t+s_{r}(t)\right)-G_{r}\left(c t-s_{r}(t)\right)=c^{-1} \hat{\sigma}_{r}\left(s_{r}(t) / t\right) .
\end{aligned}
$$

Similarly, we have boundary conditions in cases (a), (b) for $v, \sigma$ at the relief wave $x=s_{l}(t)$, which become conditions on $F_{l}, G_{l}, s_{l}$ :

$$
\begin{aligned}
& F_{l}\left(c t+s_{l}(t)\right)+G_{l}\left(c t-s_{l}(t)\right)=\hat{v}_{l}\left(s_{l}(t) / t\right), \\
& F_{l}\left(c t+s_{l}(t)\right)-G_{l}\left(c t-s_{l}(t)\right)=c^{-1} \hat{\sigma}_{l}\left(s_{l}(t) / t\right) .
\end{aligned}
$$

In case (c), for which $s_{l}(t)=-c t$, there is no rarefaction wave on the left, only an elastic shock. In this case, rye can set $\hat{v}_{l} \equiv \bar{v}_{l}, \hat{\sigma} \equiv \sigma_{r}^{0}$ in Eqs. (4.16) (where $\bar{v}_{l}, \sigma_{r}^{0}$ are defined in Sec. 4.2 under case (c)). These equations immediately determine $F_{l}(0)$ and $G_{l}$, which are constant:

$$
F_{l}(0)=\left(\bar{v}_{l}+c^{-1} \sigma_{r}^{0}\right) / 2 \text { and } G_{l}(t)=\left(\bar{v}_{l}-c^{-1} \sigma_{r}^{0}\right) / 2, \quad \text { in case (c). }
$$

REMARK. The functions $\hat{\sigma}_{l}, \hat{\sigma}_{r}$ are both given by formula (3.9) for $\hat{\sigma}$, but in general, the values of $v_{0}, \sigma_{0}, \tau_{0}$ will be different on the left and right. This dependence on the constants in (3.9) is indicated by the use of subscripts on $\hat{\sigma}_{l}, \hat{\sigma}_{r}$.

Finally, we have the boundary conditions at the shear band, which lies on the $t$-axis. By continuity of $\sigma$, and Eq. (4.2), we have

$$
\sigma_{l}(0, t)=\sigma_{r}(0, t)=b\left(\frac{c^{2} h_{1}}{\delta} \int_{0}^{t}[v](\theta) d \theta\right)
$$

where $[v](\theta)=v_{r}(0, \theta)-v_{l}(0, \theta)$. In terms of $F_{l, r}, G_{l, r}$ these boundary conditions are:

$$
\begin{aligned}
& F_{l}(c t)-G_{l}(c t)=F_{r}(c t)-G_{r}(c t), \\
& F_{r}(c t)-G_{r}(c t)=c^{-1} b\left(\frac{c^{2} h_{1}}{\delta} \int_{0}^{t}\left(F_{r}(c \theta)+G_{r}(c \theta)-F_{l}(c \theta)-G_{l}(c \theta)\right) d \theta\right) .
\end{aligned}
$$

5. Asymptotics of the symmetric case. In this section, we derive the asymptotic form of the solutions of these equations in the symmetric case. In Subsection 5.1, we work with the single variable functions $F, G, s$, and in Subsection 5.2 we deduce the form of the variables $v, \sigma, \tau, \gamma$ as functions of both $x$ and $t$.

In the symmetric case, for which the solution is shown in Figure 5(a), we may assume

$$
v_{r}^{0}=-v_{l}^{0}
$$

by translating $v$ by a constant if necessary. Then the integral curves $R_{+}$through $\left(\sigma_{r}^{0}, v_{r}^{0}\right)$ and $R_{-}$through $\left(\sigma_{l}^{0}, v_{l}^{0}\right)$ are symmetric about the $\sigma$-axis (in particular, $\left.\sigma_{l}^{0}=\sigma_{r}^{0}\right)$. Hence, we may assume that the solution in regions $E_{l}, E_{r}$ is symmetric:

$$
\sigma_{l}(x, t)=\sigma_{r}(-x, t), \quad v_{l}(x, t)=-v_{r}(-x, t), \quad s_{l}(t)=-s_{r}(t) .
$$


Therefore, we may work only with the variables in $E_{r}$, and we drop the subscripts throughout this subsection. Equations (4.15), (4.16), (4.19) reduce to three equations for the three unknown functions $F, G, s$ :

$$
\begin{aligned}
F(c t+s(t))+G(c t-s(t)) & =\hat{v}(s(t) / t), \\
F(c t+s(t))-G(c t-s(t)) & =c^{-1} \hat{\sigma}(s(t) / t), \\
F(c t)-G(c t) & =c^{-1} b\left(\frac{2 c^{2} h_{1}}{\delta} \int_{0}^{t}(F(c \theta)+G(c \theta)) d \theta\right) .
\end{aligned}
$$

Before finding the asymptotic form of the unknowns near the origin, it is convenient to process equations (5.3). We anticipate from Figure 5 that $s(t) \sim t^{1+q}$ with $q>0$. But this implies that the left-hand sides of $(5.3 \mathrm{~b}, \mathrm{c})$ coincide to leading order in $t$. We remove this degeneracy by subtracting $(5.3 \mathrm{c})$ from $(5.3 \mathrm{~b})$ :

$$
\begin{aligned}
F(c t & +s(t))-F(c t)+G(c t)-G(c t-s(t)) \\
& =c^{-1} \hat{\sigma}(s(t) / t)-c^{-1} b\left(\frac{2 c^{2} h_{1}}{\delta} \int_{0}^{t}(F(c \theta)+G(c \theta)) d \theta\right) .
\end{aligned}
$$

This processing of the equations simplifies the asymptotic analysis considerably, because as we shall see below, the leading-order terms now appear in a linearly independent set of equations. Note, however, that the leading-order powers of $t$ in Eqs. $(5.3 \mathrm{a}, \mathrm{b})$ are the same, whereas the leading order in Eq. (5.4) is higher. We could make the order of all these equations the same by dividing (5.4) by $t^{1 / 2}$, as was done for the purpose of proving short-time existence in [5]. However, this device would not simplify the asymptotic analysis. We now work with Eqs. (5.3a, b) and (5.4).

5.1. Series expansions. We seek solutions having series expansions of the form

$$
\begin{aligned}
F(t) & =F_{0}+F_{1} t^{\nu_{1}}+F_{2} t^{\nu_{2}}+\cdots, \\
G(t) & =G_{0}+G_{1} t^{\nu_{1}}+G_{2} t^{\nu_{2}}+\cdots, \\
s(t) & =s_{1} t^{\mu_{1}}+s_{2} t^{\mu_{2}}+\cdots,
\end{aligned}
$$

where the exponents $\nu_{1}<\nu_{2}, \mu_{1}<\mu_{2}$, and the coefficients $F_{i}, G_{i}, s_{i}$ are to be determined, subject only to $\nu_{1}>0, \mu_{1}>1, s_{1}>0$. We remark that we have anticipated that the expansions for $F(t)$ and $G(t)$ will involve the same powers of $t$; this is easily verified by allowing the possibility of different powers of $t$.

We shall use the expansions $(3.11 \mathrm{~b}, \mathrm{c})$ of $\hat{v}$ and $\hat{\sigma}$ and the expansion of Lemma 4.1 for $b(y)$, which we write as

$$
b(y)=\sigma_{0}-b_{2} y^{2}+O\left(y^{3}\right),
$$

with

$$
b_{2}=\alpha^{2} /\left(2 h_{1}\right)>0 .
$$

Using these expansions, we express Eqs. $(5.3 \mathrm{a}, \mathrm{b})$ and $(5.4)$ as power series about $t=0$. We describe the results of this procedure but leave the detailed expansions to be verified by the reader. The leading-order terms in $(5.3 \mathrm{a}, \mathrm{b})$ are constant, specifically

$$
F_{0}+G_{0}=v_{0}, \quad F_{0}-G_{0}=c^{-1} \sigma_{0},
$$


so that

$$
F_{0}=\frac{1}{2}\left(v_{0}+c^{-1} \sigma_{0}\right), \quad G_{0}=\frac{1}{2}\left(v_{0}-c^{-1} \sigma_{0}\right) .
$$

Continuing to equate coefficients of powers of $t$, we have the following result.

Proposition 5.1. A solution of Eqs. (5.3) admits a formal expansion in powers of $t^{1 / 2}$. This expansion is of the form (5.5), where the exponents are

$$
\mu_{1}=\nu_{1}=3 / 2, \quad \mu_{2}=\nu_{2}=2
$$

and the coefficients are given by (5.8) and formulae (5.17), (5.22) below.

Proof. Examining the lowest-order nonconstant terms in (5.3a), we have terms of order $t^{\nu_{1}}$ on the left-hand side of $(5.3 \mathrm{a})$ and $t^{3\left(\mu_{1}-1\right)}$ on the right-hand side. For a nontrivial solution, these terms must balance:

$$
\nu_{1}=3\left(\mu_{1}-1\right) \text {. }
$$

Equating coefficients at this order in $(5.3 \mathrm{a}, \mathrm{b})$, we have

$$
\begin{aligned}
& F_{1}+G_{1}=v_{3}\left(s_{1}\right)^{3} c^{-3 / 2}, \\
& F_{1}-G_{1}=0 .
\end{aligned}
$$

The third equation for $F_{1}, G_{1}, s_{1}$, and the determination of $\nu_{1}$, come from the lowest-order terms in (5.4). Here, the $t^{\nu_{1}}$ and $t^{\nu_{2}}$ terms cancel, and we have competing lowest-order terms $t^{\mu_{1}-1+\nu_{1}}$ on the left-hand side and $t^{4\left(\mu_{1}-1\right)}, t^{2}$ on the righthand side. From (5.13), we find $\mu_{1}-1+\nu_{1}=4\left(\mu_{1}-1\right)=4 \nu_{1} / 3$. If this exponent is less than two, then equating coefficients leads to the conclusion that $F_{1}, G_{1}, s_{1}$ must all be zero. On the other hand if $4 \nu_{1} / 3>2$, then we reach a contradiction because the coefficient of $t^{2}$ is nonzero. We conclude

$$
\begin{aligned}
\nu_{1} & =3 / 2, \\
\frac{3}{2} c^{1 / 2} F_{1} s_{1}+\frac{3}{2} c^{1 / 2} G_{1} s_{1} & =-c^{-1} \sigma_{4}\left(s_{1}\right)^{4}+4 c^{3} b_{2} h_{1}^{2} v_{0}^{2} / \delta^{2} .
\end{aligned}
$$

Solving (5.11)-(5.14), we obtain

$$
\begin{gathered}
\nu_{1}=\mu_{1}=3 / 2 \\
F_{1}=G_{1}=c^{-3 / 2} v_{3} s_{1}^{3} / 2, \quad s_{1}=\left(\frac{8 b_{2} h_{1}^{2} v_{0}^{2}}{\delta^{2}\left(3 v_{3}+2 \sigma_{4}\right)}\right)^{1 / 4} c .
\end{gathered}
$$

Using expressions (3.12) for $v_{3}, \sigma_{4}$ and (5.7) for $b_{2}$, we get

$$
F_{1}=G_{1}=\frac{1}{3}\left(\frac{4}{3}\right)^{3 / 4} \alpha h_{1}^{1 / 2} c^{1 / 2}\left(\frac{v_{0}}{\delta}\right)^{3 / 2}, \quad s_{1}=\left(\frac{4}{3}\right)^{1 / 4} \alpha c^{2} h_{1}^{1 / 2}\left(\frac{v_{0}}{\delta}\right)^{1 / 2} \text {. }
$$

Now we examine the next-order terms. In fact, we need these in the next subsection to examine the dependence of $v$ and $\sigma$ on $(x, t)$. Balancing powers of $t$ and arguing as above, we find from $(5.3 \mathrm{a}, \mathrm{b})$ that the next-order terms occur at order $t^{2}$. This leads to the determination of $\nu_{2}, \mu_{2}$ in (5.5):

$$
\nu_{2}=\mu_{2}=2 \text {, }
$$


and to two of the three equations for coefficients $F_{2}, G_{2}, s_{2}$ :

$$
\begin{aligned}
c^{2}\left(F_{2}+G_{2}\right) & =3 v_{3}\left(s_{1}\right)^{2} s_{2}, \\
3 c^{1 / 2} F_{1} s_{1}+c^{2}\left(F_{2}-G_{2}\right) & =-\sigma_{4}\left(s_{1}\right)^{4} / c .
\end{aligned}
$$

The next-order terms in (5.4) are at order $t^{5 / 2}$, leading to the third equation for the coefficients:

$$
3 c^{1 / 2} F_{1} s_{2}+2 c s_{1}\left(F_{2}+G_{2}\right)=-4 \sigma_{4}\left(s_{1}\right)^{3} s_{2} / c .
$$

(It might appear that the next-order terms (i.e., order $t^{5 / 2}$ ) in $F$ and $G$ would contribute to (5.4) at the level of (5.20), but in fact these terms cancel.)

To solve (5.19), (5.20), we first substitute for $F_{1}$ in terms of $s_{1}$ using (5.16) and write the system in matrix form:

$$
\left(\begin{array}{ccc}
1 & 1 & -3 c^{-2} v_{3}\left(s_{1}\right)^{2} \\
1 & -1 & 0 \\
2 c s_{1} & 2 c s_{1} & \left(4 \sigma_{4}+\frac{3}{2} v_{3}\right)\left(s_{1}\right)^{3} / c
\end{array}\right)\left(\begin{array}{c}
F_{2} \\
G_{2} \\
s_{2}
\end{array}\right)=\left(\begin{array}{c}
0 \\
-\left(s_{1}\right)^{4} c^{-3}\left(\sigma_{4}+\frac{3}{2} v_{3}\right) \\
0
\end{array}\right) .
$$

The matrix on the left-hand side of (5.21) has determinant $-2\left(s_{1}\right)^{3}\left(4 \sigma_{4}+\frac{15}{2} v_{3}\right) / c$, which is nonzero. Solving (5.21), we have

$$
\left(\begin{array}{c}
F_{2} \\
G_{2} \\
s_{2}
\end{array}\right)=\frac{1}{2}\left(s_{1}\right)^{4} c^{-3}\left(\sigma_{4}+\frac{3}{2} v_{3}\right)\left(\begin{array}{c}
-1 \\
1 \\
0
\end{array}\right)
$$

This completes the proof of Proposition 5.1.

5.2. Behavior of solutions. Now we can deduce the dependence of the variables $v, \sigma, \tau, \gamma$ on $(x, t)$ in the region $0 \leq x \leq s(t)$ for small $t$. In Figure 6 we graph the functions $v(x, t), \sigma(x, t), \tau(x, t), \gamma(x, t)$ as functions of $x$ at fixed time $t$, over an interval that overlaps the rarefaction waves. The blown-up solutions of Figure 4 are shown in Figure 6 as dotted curves, and the solutions of Figure 5 are shown as solid curves. These agree outside the region $|x|<s(t)$ bounded by the relief waves, since the effect of the shear band is not felt ahead of the relief wave. The graphs in Figure 6 are based on the following proposition.

Proposition 5.2. For $0<x<s(t)$, and near $t=0$, the functions have the following expansions, uniform in $x$ :

$$
\begin{aligned}
& v(x, t)=v_{0}+2 c A t^{3 / 2}+O\left(t^{5 / 2}\right), \\
& \sigma(x, t)=\sigma_{0}+3 c^{1 / 2} A x t^{1 / 2}+B t^{2}+O\left(t^{5 / 2}\right), \\
& \tau(x, t)=\tau_{0}-\frac{s_{1}^{4 / 3}}{h_{1} \alpha} x^{2 / 3}+O\left(t^{2}\right), \\
& \gamma(x, t)=-\frac{s_{1}^{4 / 3}}{h_{1}} x^{2 / 3}+O\left(t^{2}\right),
\end{aligned}
$$

where

$$
\begin{aligned}
& A=\frac{1}{3}\left(\frac{4}{3}\right)^{3 / 4} \alpha h_{1}^{1 / 2} c^{1 / 2}\left(\frac{v_{0}}{\delta}\right)^{3 / 2}>0, \\
& B=-s_{1}^{4}\left(\sigma_{4}+\frac{3}{2} v_{3}\right)=-2 \alpha^{2} h_{1} c^{4}\left(\frac{v_{0}}{\delta}\right)^{2}<0 .
\end{aligned}
$$



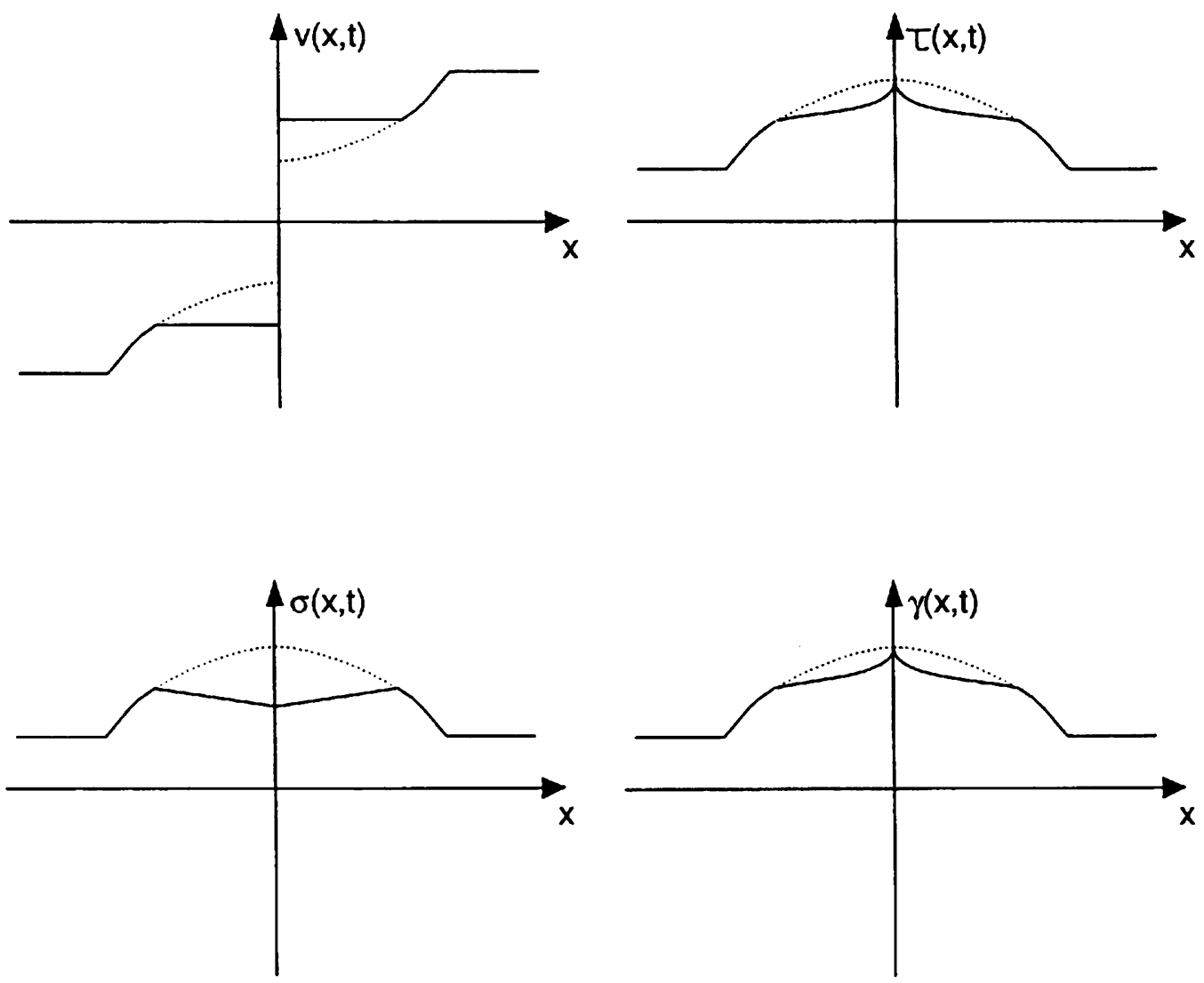

FIG. 6. Graphs of the solution (solid) and the blown-up solution (dots)

Proof. Using the fact that $x \leq s(t)=O\left(t^{3 / 2}\right)$ and retaining terms up to order $t^{2}$, we have

$$
\begin{aligned}
(c t+x)^{3 / 2}+(c t-x)^{3 / 2} & =2 c t^{3 / 2}+O\left(t^{5 / 2}\right), \\
(c t+x)^{3 / 2}-(c t-x)^{3 / 2} & =3 c^{1 / 2} x t^{1 / 2}+O\left(t^{5 / 2}\right), \\
(c t+x)^{2}+(c t-x)^{2} & =2 c^{2} t^{2}+O\left(t^{5 / 2}\right), \\
(c t+x)^{2}-(c t-x)^{2} & =O\left(t^{5 / 2}\right) .
\end{aligned}
$$

A short calculation yields the formulae $(5.23 \mathrm{a}, \mathrm{b})$.

Now $\tau$ and $\gamma$ are both independent of $t$ for $0 \leq x \leq s(t)$, so that

$$
\begin{aligned}
\tau(x, t) & =\hat{\tau}(x / \hat{t}(x)), \\
\gamma(x, t) & =\hat{\gamma}(x / \hat{t}(x)),
\end{aligned}
$$

where $\hat{t}=\hat{t}(x)$ satisfies

$$
s(\hat{t})=x
$$


Using $s(t) \sim s_{1} t^{3 / 2}+O\left(t^{2}\right)$, we obtain from (5.27),

$$
\hat{t}(x) \sim\left(\frac{x}{s_{1}}\right)^{2 / 3}+O(x) .
$$

Now using the asymptotic form (3.11d), (3.11a) for $\hat{\tau}$ and $\hat{\gamma}$, we obtain the formulae $(5.23 \mathrm{c}, \mathrm{d})$. The proof is complete.

Note that in the elastic region $0<x<s(t)$, the functions $\tau, \gamma$ are independent of $t$. Note also that we have continued the expansion of these functions to a lower order than those of $v$ and $\sigma$ because of the order $t^{2}$ terms in the latter functions have clear significance whereas the order $x^{4 / 3}$ (i.e., order $t^{2}$ terms) have less significance in $\tau$ and $\gamma$.

Proposition 5.2 shows that, up to order $t^{2}, v$ is independent of $x$ and is increasing in time, whereas $\sigma$ is linear in $x$, with positive slope, but is decreasing in time. (The function $\sigma$ is clearly decreasing at $x=0$; at $x=s(t)$, we have $\sigma=\hat{\sigma}(s(t) / t)$, which is decreasing.)

Appendix. Derivation of equations. In this appendix, we derive Eqs. (2.1) from the equations proposed in [4] to model antiplane shearing. This is achieved by (i) restricting to solutions depending on only one space variable and (ii) linearizing the yield condition at an appropriately chosen point consistent with the restriction to one space dimension. These simplifications of the equations for one-dimensional solutions do not affect the leading asymptotic behavior. We make the simplifications in order to obtain simple expressions for the variables in rarefaction waves and to simplify the process (in Sec. 4.1) of integrating the equations for evolution of the variables in a shear band.

Recall from [4], the equations of the model for antiplane shearing:

$$
\begin{array}{rlrl}
\partial_{t} v & =\operatorname{div} T \\
{\left[I+\frac{1}{h(|T|)}(R T) T^{\mathrm{T}}\right] \partial_{t} T} & =c^{2} \operatorname{grad} v & & \text { (if loading) } \\
\partial_{t} T & =c^{2} \operatorname{grad} v & & \text { (if unloading) } .
\end{array}
$$

The dependent variables $v$ and $T=\left(T_{1}, T_{2}\right)$ depend on spatial variables $x=$ $\left(x_{1}, x_{2}\right)$ and time $t$; they represent velocity (in the $x_{3}$-direction) and stress, respectively, where $T_{j}$ is the $T_{3 j}$ component of the full stress tensor, $j=1,2$, all other entries $T_{i j}$ being constant. $T^{\mathrm{T}}$ is the transpose of $T$. The constant $c$ is the elastic wave speed, $I$ is the $2 \times 2$ identity matrix, and $R$ is the rotation matrix

$$
R=\left(\begin{array}{cc}
\cos \beta & \sin \beta \\
-\sin \beta & \cos \beta
\end{array}\right)
$$

with parameter $\beta \in\left(0, \frac{\pi}{2}\right)$ measuring the degree of nonassociativity in the model (specifically in the flow rule). The given function $h=h(|T|)$ is the hardening modulus. It is a nonnegative monotonically decreasing function on $[0,1]$ with $h(1)=0$.

Equation (A.1a) is conservation of momentum, while Eqs. (A.1b, c) specify constitutive behavior. This behavior is described as loading (or plastic) when $|T|$ is at 
its maximum over previous time and is increasing, i.e., the material is loading when it is at plastic yield:

$$
|T(x, t)|=\max _{0 \leq s \leq t}|T(x, s)|,
$$

and the right-hand side of (A.3) is increasing:

$$
\partial_{t}|T(x, t)|>0 \text {. }
$$

Otherwise, the material behavior is said to be unloading (or elastic).

For the purpose of this paper, it is convenient to introduce the yield stress $\gamma$ :

$$
\gamma(x, t)=\max _{0 \leq s \leq t}|T(x, s)| .
$$

Then the yield condition (A.3) becomes

$$
|T(x, t)|=\gamma(x, t),
$$

and we have a differential equation for $\gamma$ :

$$
\partial_{t} \gamma= \begin{cases}\partial_{t}|T| & \text { if loading } \\ 0 & \text { if unloading. }\end{cases}
$$

In this paper, we consider solutions of (A.1) depending on one space variable, say $x_{1}$. It was shown in [4] that there are uniform solutions

$$
v=x_{1}, \quad T=T(t)
$$

and that these solutions eventually lose stability (in fact, the equations become illposed) to perturbations by plane waves in some direction. In [4], it is shown that there is a number $\gamma_{B}, 0<\gamma_{B}<1$, defined by

$$
h\left(\gamma_{B}\right)=\gamma_{B}^{2} \sin ^{2} \frac{\beta}{2}
$$

with the property that Eqs. (A.1) in the loading case are hyperbolic if $|T|<\gamma_{B}$ and nonhyperbolic otherwise.

Although uniform solutions lose stability to plane wave perturbations in some direction, only for special choices of initial conditions do the uniform solutions first lose stability to plane wave perturbations in the same direction, parallel to the $x_{1}$-axis. Only for this special family of solutions can the solution remain one-dimensional and physical. In this case, the equations lose hyperbolicity when

$$
T=\gamma_{B}\left(\cos \frac{\beta}{2}, \sin \frac{\beta}{2}\right) \text {. }
$$

(See [4] for further details regarding the loss of stability of uniform solutions.)

We approximate Eqs. (A.1) (for one-dimensional solutions) by linearizing the yield condition at $T=T_{B}$ given by the right-hand side of (A.10) and evaluating $(R T) T^{\mathrm{T}}$ at $T=T_{B}$. However, we retain nonlinear dependence in the loading equations by keeping the full dependence of $h(\gamma)$ on $\gamma$.

Let $\gamma=\gamma_{B}+\gamma^{\prime}, T=T_{B}+\widetilde{T}$. Linearizing the yield condition $\gamma=|T|$ at $\gamma=\gamma_{B}$, $T=T_{B}$, we obtain

$$
\gamma^{\prime}=\widetilde{T}_{1} \cos \frac{\beta}{2}+\widetilde{T}_{2} \sin \frac{\beta}{2} .
$$


Define

$$
\tilde{\gamma}=\widetilde{T}_{1}+\alpha \widetilde{T}_{2}, \quad \text { where } \alpha=\tan \frac{\beta}{2}
$$

Then

$$
\left[\left(R T_{B}\right) T_{B}^{\mathrm{T}}\right] \partial_{t} T=\gamma_{B}^{2} \cos ^{2} \frac{\beta}{2}\left(\begin{array}{c}
1 \\
-\alpha
\end{array}\right) \partial_{t} \tilde{\gamma} .
$$

Next, replace $h(\gamma)$ by a new function

$$
\tilde{h}(\tilde{\gamma})=\gamma_{B}^{-2} \sec ^{2} \frac{\beta}{2} h\left(\gamma_{B}+\tilde{\gamma} \cos \frac{\beta}{2}\right) .
$$

Then $\tilde{h}(\tilde{\gamma})$ is a positive decreasing function on an interval $\left[\gamma_{1}, \gamma_{2}\right], \gamma_{1}<0<\gamma_{2}$, and from (A.9) we find

$$
\tilde{h}(0)=\alpha^{2} \text {. }
$$

Finally, we relabel $x_{1}$ as $x, \widetilde{T}_{1}$ as $\sigma$, and $\widetilde{T}_{2}$ as $\tau$ and drop the tildes on $\tilde{h}$ and $\tilde{\gamma}$. Then our approximation to Eqs. (A.1) for solutions depending on one space dimension becomes system (2.1).

Acknowledgment. We are grateful to Xabier Garaizar and John Trangenstein for helpful discussions related to this paper.

\section{REFERENCES}

[1] X. Garaizar, Numerical computations for antiplane shear in a granular flow model, Quart. Appl. Math. 52, 289-309 (1994)

[2] X. Garaizar and D. G. Schaeffer, Numerical computations for shear bands in an antiplane shear model, J. Mech. Phys. Solids 42, 21-50 (1994)

[3] P. D. Lax, Hyperbolic systems of conservation laws. II, Comm. Pure Appl. Math. 10, 537-566 (1957)

[4] D. G. Schaeffer, A mathematical model for localization in granular flow, Proc. Roy. Soc. London Ser. A 436, 217-250 (1992)

[5] D. G. Schaeffer and M. Shearer, Unloading near a shear band: A free boundary problem for the wave equation, Comm. Pure Appl. Math. 18, 1271-1298 (1993) 